\title{
3D Structure Studies of the Pancreatic Beta Cell by High Resolution Cellular Tomography
}

\author{
B. J. Marsh*, D. N. Mastronarde*, K. E. Howell**, J. C. Hutton***, C. J. Rhodes**** and J. R. \\ McIntosh*
}

*Boulder Laboratory for 3D EM, Department of Molecular, Cellular and Developmental Biology, University of Colorado, Boulder, CO 80309, **Dept. of Cellular and Structural Biology, University of Colorado School of Medicine, Denver, CO 80262, ***Barbara Davis Center for Childhood Diabetes, University of Colorado Health Sciences Center, Denver, CO 80262, ****Pacific Northwest Research Institute, 720 Broadway, Seattle, WA 98122

We have used high-pressure freezing, freeze-substitution and dual-axis electron microscope (EM) tomography to study the organelles involved in the synthesis, processing and secretion of insulin in three dimensions (3D) at $\sim 6 \mathrm{~nm}$ resolution in the pancreatic beta cell line HIT-T15. These data have: (1) revealed the complexity of structural relationships among the Golgi, endoplasmic reticulum (ER) and compartments of the endosomal-lysosomal system [1], (2) provided evidence that multiple transport mechanisms act in concert in the same region of the Golgi ribbon [2], and (3) suggested a role for the ER in regulating membrane traffic/sorting at the trans Golgi - the presumptive site where proinsulin is sorted and packaged into nascent secretory granules $[1,2]$.

We are now using these methods to analyze mechanisms related to insulin granule biogenesis, movement and recruitment to sites of exocytosis in islets of Langerhans isolated from Balb/c mice. 250-400nm-thick sections cut from high-pressure frozen, freeze-substituted and plastic-embedded islets are imaged either at $300 \mathrm{keV}$ on a Technai F30 intermediate voltage EM (FEI) or at $750 \mathrm{keV}$ on a JEM-1000 high voltage EM (JEOL) using high tilt-rotate specimen holders (Gatan). Tilt series data are recorded digitally as the sections are serially tilted through $1^{\circ}$ or $1.5^{\circ}$ increments over a range of $140^{\circ}$ or $120^{\circ}$, respectively, about two orthogonal axes using the microscope control program SerialEM. Semi-automated methods for CCD data acquisition, image montaging and image alignment by cross-correlation controlled by SerialEM help to minimize electron dose to the specimen. Images are more accurately aligned with one another by tracking the positions of gold fiducial markers $(10-15 \mathrm{~nm})$ on both surfaces of the sections using a modification of the TILTALIGN program that uses subsets of fiducials to solve for local alignments, and movies of the aligned "tilt series" images are constructed [3]. The 3D density distributions (tomograms) calculated using an Rweighted back-projection program from each set of aligned tilts are then aligned with each other and combined to produce a single, dual-axis 3D reconstruction [4] (FIG. 1).

$3 \mathrm{D}$ reconstructions (each measuring at least $4 \square \mathrm{m} \times 4 \square \mathrm{m} \times 0.3 \square \mathrm{m}$ ) generated in this way and modeled using the IMOD software package [5] reveal the structural complexity of processing compartment intermediates at the trans-face of the Golgi (FIG. 2), and provide further evidence that cargo is sorted not in one but in multiple, distinct trans-cisternae. These data also continue to support a role for the ER in sorting and packaging cargo for exit and/or modifying the lipid composition of trans-Golgi cisternal membranes.

To lay the groundwork for visualizing these organelles in a close-to-native state in $3 \mathrm{D}$, we have initiated: (1) cryo-EM tomography of insulin granules and stacked Golgi fractions isolated from the 
insulin secretory pathway of insulinoma beta cells and embedded in a thin layer of vitreous ice by plunge-freezing (FIG. 3), and (2) cutting frozen-hydrated sections directly from high-pressure frozen, vitrified mouse islets at $-170^{\circ} \mathrm{C}$ (FIG. 4). 3D reconstructions generated from such specimens should allow us to relate 3D structure data at the subcellular and molecular levels to the biochemical mechanisms for insulin granule formation and exocytosis in the pancreatic beta cell.

\section{References}

[1] B. J. Marsh et al., Proc. Natl. Acad. Sci. USA 98 (2001) 2399.

[2] B. J. Marsh et al., Biochem. Soc. Trans. 29 (2001) 461.

[3] D. N. Mastronarde et al., Microsc. Microanal. 7 (Suppl. 2) (2001) 90.

[4] D. N. Mastronarde, J. Struct. Biol. 120 (1997) 343.

[5] J. R. Kremer et al., J. Struct. Biol. 116 (1996) 71.

[6] This work was supported by Juvenile Diabetes Research Foundation International Postdoctoral Fellowship award 3-1999-538 to B. J. M., NIH/NCRR grant RR00592 to J. R. M., and NIH Program Project grant P01GM61306 to K. E.H. and J. R.M.
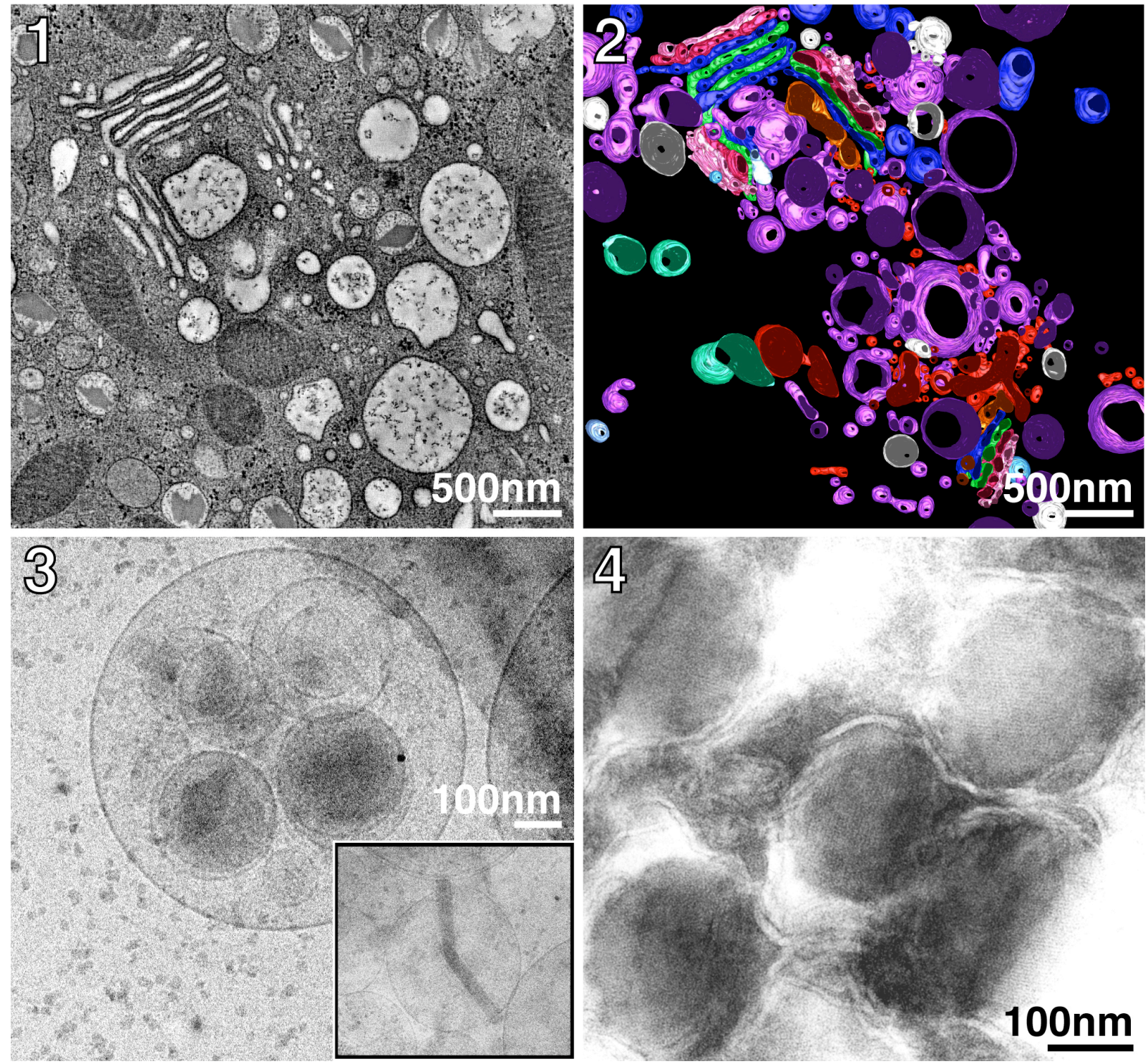